\title{
Improved Detection of Probe Request Attacks Using Neural Networks and Genetic Algorithm
}

\author{
Deepthi N. Ratnayake ${ }^{1}$, Hassan B. Kazemian ${ }^{1}$ and Syed A. Yusuf ${ }^{2}$ \\ ${ }^{1}$ Intelligent Systems Research Centre, Faculty of Computing, London Metropolitan University, \\ 166-220 Holloway Road, N7 8DB, London, U.K. \\ ${ }^{2}$ Institute of Industrial Research, University of Portsmouth, PO1 2EG, Portsmouth, U.K.
}

\begin{abstract}
Keywords: Wlan Security, Probe Request Flooding Attacks, Neural Networks, Genetic Algorithms.
Abstract: $\quad$ The Media Access Control (MAC) layer of the wireless protocol, Institute of Electrical and Electronics Engineers (IEEE) 802.11, is based on the exchange of request and response messages. Probe Request Flooding Attacks (PRFA) are devised based on this design flaw to reduce network performance or prevent legitimate users from accessing network resources. The vulnerability is amplified due to clear beacon, probe request and probe response frames. The research is to detect PRFA of Wireless Local Area Networks (WLAN) using a Supervised Feedforward Neural Network (NN). The NN converged outstandingly with train, valid, test sample percentages $70,15,15$ and hidden neurons 20 . The effectiveness of an Intruder Detection System depends on its prediction accuracy. This paper presents optimisation of the NN using Genetic Algorithms (GA). GAs sought to maximise the performance of the model based on Linear Regression $(\mathrm{R})$ and generated $\mathrm{R}>0.95$. Novelty of this research lies in the fact that the NN accepts user and attacker training data captured separately. Hence, security administrators do not have to perform the painstaking task of manually identifying individual frames for labelling prior training. The GA provides a reliable NN model and recognises the behaviour of the $\mathrm{NN}$ for diverse configurations.
\end{abstract}

\section{INTRODUCTION}

Wireless networks are springing up everywhere as it enables an information-rich environment through computers and handheld devices with portability and flexibility, increased productivity, and lower installation costs. However, the openly exposed airwave signals, by their nature have increased risks in security. Wireless Local Area Network (WLAN) management frames facilitate stations to begin and maintain connections. In infrastructure WLANs, any wireless station with a legitimate Media Access Control (MAC) address can send a probe request management frame when it needs information from an Access Point (AP). AP replies to any probe request with a probe response management frame with capability information, such as the network name access point name, supported data rates, etc. Probe request and response management frames are open, so the information can be obtained using freely available sniffing software. Almost all Wireless Network Interface Cards (NICs) permit changing their MAC addresses to arbitrary values through vendor-supplied/open-source drivers or an application program. Probe Request Flooding Attacks (PRFA) send a burst of probe requests, having different source MAC addresses to generate a heavy workload on the AP which can lead to a Denial-of-Service (DoS) to its legitimate users. Vulnerability arising from PRFA can also be exploited to create other advanced attacks.

In a real WLAN, traffic pattern is usually unpredictable and also varies on the usage, operating system and applications used. Further, Wireless Intrusion Detection System (WIDS) may fail to capture some of the frames due to network or environmental issues. Neural Networks (NN) considered ideal in dealing with real-world tasks, where data is often messy, uncertain or inconsistent and perfect solutions may not exist. The capacity to learn by example makes NNs exceptionally flexible and powerful as there is no need to create algorithms to execute a task. The parallel architecture facilitates high response and computational times. These make NNs a perfect candidate for application of wireless attack detection in real world complex environments 
(Karygiannis and Owens, 2002); (Landau and Taylor, 1997); (Ratnayake et al., 2011); (Zhang et al., 2008)

In a previous empirical study by the authors (Ratnayake et al., 2011), an external attacker was identified by analysing the traffic generated from a user MAC address in a single frequency band of a WLAN. A supervised feed-forward NN with four distinct inputs, delta-time, sequence number, signal strength and frame sub-type is applied to identify and differentiate a genuine frame from a rogue frame. The experimental results showed that the use of NN can detect probe request attacks to a very high precision.

However, the effectiveness of an Intrusion Detection System (IDS) is evaluated by its ability to make correct predictions, and therefore, the $\mathrm{NN}$ has to be optimised and trained. NN optimisation is the art and science of arranging interconnected factors to the best possible effect. Many methods are in use including trial-and-error, which are not rigorous enough to arrive at a truly optimal structure. In contrast, Genetic Algorithms (GA) outperform traditional optimisation methods, due to its rigorousness in finding optimal parameters. GA is a search heuristic that is modelled on the principles of natural selection, reproduction by crossover and mutation. A fitness function is applied to evaluate individuals. GA was first used by (Holland, 1975). (De Jong, 1993) established that population-based GAs using crossover and mutation could successfully be applied to optimisation problems in many areas. Some research work based on GA in the field of intrusion detection are presented in (Bankovic et al., 2007); (Gong et al., 2005); (Haddadi et al., 2010); (Hua and Xiaofeng, 2008); (Li, 2004); (Pillai et al., 2004); (Wu and Banzhaf, 2010); (Xia et al., 2005); (Yao, 2010). GAs are frequently successful and considered as the most popular in real applications (Reeves and Rowe, 2003).

This paper discusses the structural optimisation component of the NN designed to detect probe request attacks, using GA. The paper is organised as follows: Section 2 - Probe request attack detection using neural networks, Section 3 - Neural network structure optimisation, Section 4 - Results and discussion, and Section 5 - Conclusion.

\section{PROBE REQUEST ATTACK DETECTION}

A supervised feed-forward $\mathrm{NN}$ was designed with 4 input neurons, one hidden layer, with 20 hidden neurons and a linear output neuron which classifies genuine frames from rogue frames. The initial design divided the data sample and used $70 \%$ of the data to train the network and $15 \%$ each for validation and testing respectively. LevenbergMarquardt back propagation algorithm was utilised for training (Ratnayake et al., 2011). The results are shown in Table 1. Performance of the network was determined by the overall regression value.

Table 1: Results from the Un-optimised NN.

\begin{tabular}{lr}
\hline Training Sample 70\% & 123640 \\
Validation Sample 15\% & 26495 \\
Testing Sample 15\% & 26495 \\
Hidden Neurons & 20 \\
Epoch & 77 \\
Performance & 0.00386 \\
(The Mean Squared Errors (MSEs) of training, validation and \\
testing were 3.86409e-3,3.75033e-3 and 3.67129e-3) \\
Regression (overall) & 0.98043 \\
\hline
\end{tabular}

However, NN structural-values were $70 / 15 / 15$ sample sizes and one hidden layer with 20 neurons. The model's performance can be misleading due to over-fitting, which generally occurs when the model is not evaluated during the NN training. Over-fitted models do not perform well on unseen data. Crossvalidation is among several methods of estimating how well a trained model will perform with unseen data and detect and prevent over-fitting of the model (Moore, 2001). A 5-fold cross validation test was performed using the sample data set, and the results showed that the trained $\mathrm{NN}$ will perform considerably well with unseen data with 73.59 to $100 \%$ accuracy rate. However, the intention of this research is to realise a $100 \%$ accurate $\mathrm{NN}$ model.

\section{NEURAL NETWORK OPTIMISATION}

NN performance can be improved by initialising the network and training, increasing the number of hidden neurons and layers, using a different training function, or using additional training data (MathWorks, 2012).

This research uses GA approach to optimise the constructs of NN structure, namely; training, validation and testing sample percentages, and number of neurons of the hidden layer. The training sample contains approximately $175 \mathrm{~K}$ frames. Larger numbers of neurons and extra hidden layers give the NN more flexibility since network has more 


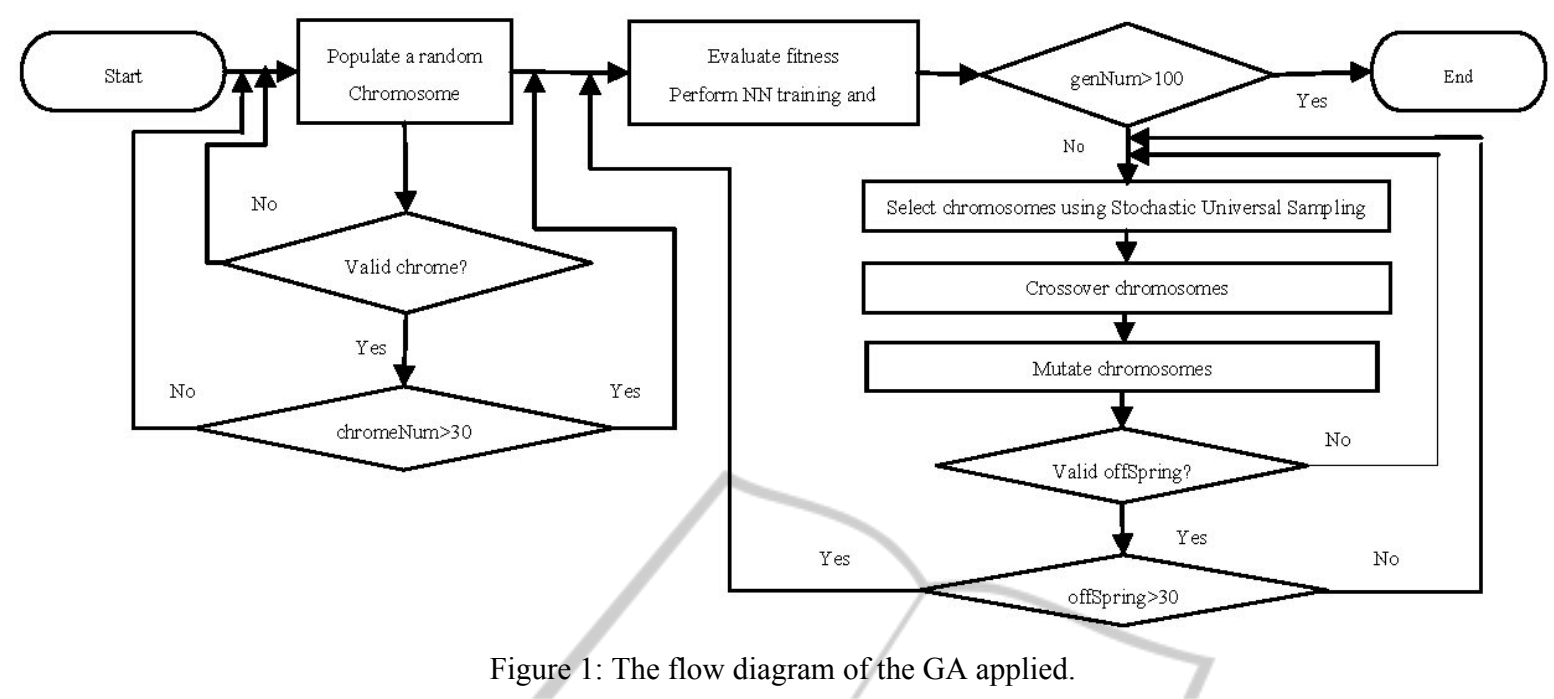

parameters for optimisation. However, a too large hidden layer can cause the problem to be undercharacterised and the network must optimise more parameters than there are data vectors to constrain these parameters. Further, more neurons and layers increase processing time, and demands high processing power (MathWorks, 2012). Therefore, in this experiment, a single hidden layer and maximum of 32 hidden neurons were used. A single training algorithm is selected to train the network. A mix of MATLAB and Netbeans/JGAP environment were used to optimise the solution.

(Reeves and Rowe, 2003) state that probably everybody's GA is unique. Although, GAs can find global optima, when it is applied to different problems, there are other attractors to which they may converge, with a distance from global optimality. Since Holland and De Jong, researchers and practitioners have recommended many variations of GA recommending population sizes, initialisation methods, fitness definitions, selection and replacement strategies, and crossover and mutation methods. However, Practitioner's viewpoint, (Levine, 1997) has a clear and reasonable deviation from traditional GAs, which this research utilised as a guideline.

The flow diagram of GA applied is shown in Figure 1. Population of random chromosomes, validation, crossover and mutation were performed in Netbeans Environment. NN training, calculation of fitness and fitness scaling were performed in MATLAB environment. The algorithm ran 3 times with different selection and mutation configurations.

Population created a population of a binary vector that represents 4 genes (Table 2). Function randomGeneration created the initial population, and crossOver and mutation functions created the populations of the rest of the generations. A validation function validated genes. Therefore, population is biased to create a chromosome with genes that are on the boundaries of the constraints, and to create well-dispersed populations. The size of the population is 30 chromosomes.

Table 2: Genes of the Chromosome.

\begin{tabular}{|l|l|}
\hline Gene Name & \multicolumn{1}{|c|}{ Description } \\
\hline trainVal & \% of training sample \\
\hline validVal & \% of validation sample \\
\hline testVal & \% of testing sample \\
\hline neuL1 & number of neurons in hidden layer 1 \\
\hline
\end{tabular}

Table 3: Validation constraints.

\begin{tabular}{|l|}
\hline Sizes of trainVal, validVal, and testVal should be $>0$ and $<98$ \\
\hline Total of trainVal, validVal, testVal should be $=100$ \\
\hline neuL1 should be $>0$ and $<33$ \\
\hline
\end{tabular}

Fitness evaluation was performed as follows; Gene values of each chromosome were fed into NN model. The network used MATLAB back propagation training function - trainlm, with default training parameters. Function trainlm is considered as the fastest backpropagation algorithm in the toolbox, however, it does require more memory than other algorithms. Function trainlm updates weight and bias values according to Levenberg-Marquardt optimisation (MathWorks, 2012).

The information recorded during train, sim, and regress functions are; Regression, MSE, and best epoch. Regression analysis is a statistical tool for the examination of interaction between variables (Lindley, 1987). The MATLAB function postreg was used to calculate the fitness value $[R]$ egression. The function performs a linear regression between 
the NN output and the target, and computes the correlation coefficient ( $\mathrm{R}$ value). Stochastic Universal Sampling (SUS) was used for selecting chromosomes for recombination. SUS uses a single random value to sample all of the solutions by choosing them at evenly spaced intervals. Therefore, it offers zero bias and minimum spread (Baker, 1987). A fitness scaling was performed using MATLAB function fitscalingrank. A pre-determined $\%$ of the total population was selected for crossover using MATLAB function selectionstochunif. Single point crossovers were performed. The position of the crossover was generated randomly. The concepts of élitism and population overlaps were used in creating new population, i.e. one elite chromosome, which is the best performed chromosome from each generation, was migrated to the next generation. Selected chromosomes were crossed until 29 more valid chromosomes were generated to complete the next generation. Chromosomes to be mutated and position to be mutated were selected randomly. Mutated chromosomes were validated using conditions in Table 3. The stopping criterion for the $\mathrm{GA}$ is when maximum number of generations is reached or when there is no improvement.

The GA was set for 100 generations with 30 chromosomes in each generation, and 4 different types of genes in each chromosome. This experiment was run on a Toshiba Satellite Pro M70 laptop with an Intel ${ }^{\circledR}$ Pentium ${ }^{\circledR}$ M $740(1.73 \mathrm{GHz})$ microprocessor and 1.9 gigabytes of Random Access Memory, Microsoft Windows XP OS, and a standard JDK with MATLAB 2008b. Matlabcontrol Java API was used to interface Java and MATLAB.

\section{RESULTS AND DISCUSSION}

The GA was run several times with different parameters. The following presents 3 unique GAs that shaped the ultimate objective of the research.

The initial GA was executed up to 59 generations with maxNeurons $=25,1 \%$ of single point mutations. fitnessScale $=0.6$. To maintain mutation at $1 \%$ of total population, mutation was performed on one newly formed offspring at every 3 rd generation. The GA was stopped when there was no improvement in regression value after 42 nd generation. Minimum and maximum MSEs were 0.0035 and 0.0046 . Regression values reported were between 0.9560 and 0.9679 . GA converged at $8^{\text {th }}$ generation when trainVal, validVal, testVal and neuL1 were 21, 33, 39 and 3 respectively. When the results were analysed, it was found that insufficient chromosomes were validated to the next generation, resulting rapid diversity reduction in the population.

Therefore, in the 2nd GA, the maxNeurons were increased to 32, and fitnessScale rate to $90 \%$. The GA was executed up to 76 generations. The GA was stopped when there was no improvement in regression value after 41 st generation. Minimum and maximum MSEs were 0.0036 and 0.0045 . Regression values reported were between 0.9578 and 0.9691 . GA converged at 2nd generation when trainVal, validVal, testVal and neuL1 were 24, 06, 70 and 27 respectively. When the results were analysed, it was found that the problem of insufficient validated chromosomes to the next generation, still causes rapid diversity reduction in the population. Further analysis showed that the strict validation rules restricted achieving diversity. Although, the research used a high selection and crossover probability, the validation after crossover and mutation rejects a major percentage of new offspring migrating into the next generation. Selection, crossover and mutation play a major role in evolution, creating diversity. Therefore, in nature and in GAs, the key feature for good performance is to sustain the diversity of the population. Selection methods also can lead to reduce diversity swiftly. Therefore, the validation rules of GA were revised to accept offspring, by scaling the absolute values to relative values of $100 \%$, so that the total of trainVal+validVal+testVal will always equal to 100 . The calculations were performed using the Largest Remainder Method. SUS was not changed as there was no visible effect between $60 \%$ and $80 \%$ rates during previous tests. However, a two-point mutation was performed. Revision of maxNeurons may also have improved the diversity and results. However, current hardware constrains restrict increasing the neuL1 limit.

The GA3 was executed up to 100 generations. GA converged at 12th generation. Maximum overall regression value 0.97682 was achieved when trainVal, validVal, and testVal and neuL1 were 1, 43, 56 and 6 respectively. Minimum regression value 0.96101 was achieved when trainVal, validVal, and testVal and neuL1 were 41,39, 20 and 17 respectively. Minimum and maximum MSEs were 0.0084378 and 0.0036468 (Figure 2). The search space analysis showed a considerably good diversity in chromosomes generated by the GA.

All GAs generated impressive results with $\mathrm{R}>$ 0.95 , which is very close to the ideal fitness value of 1. This shows that the training data is comprehensive. It was achieved due to the large training sample. Obtaining a large training sample 


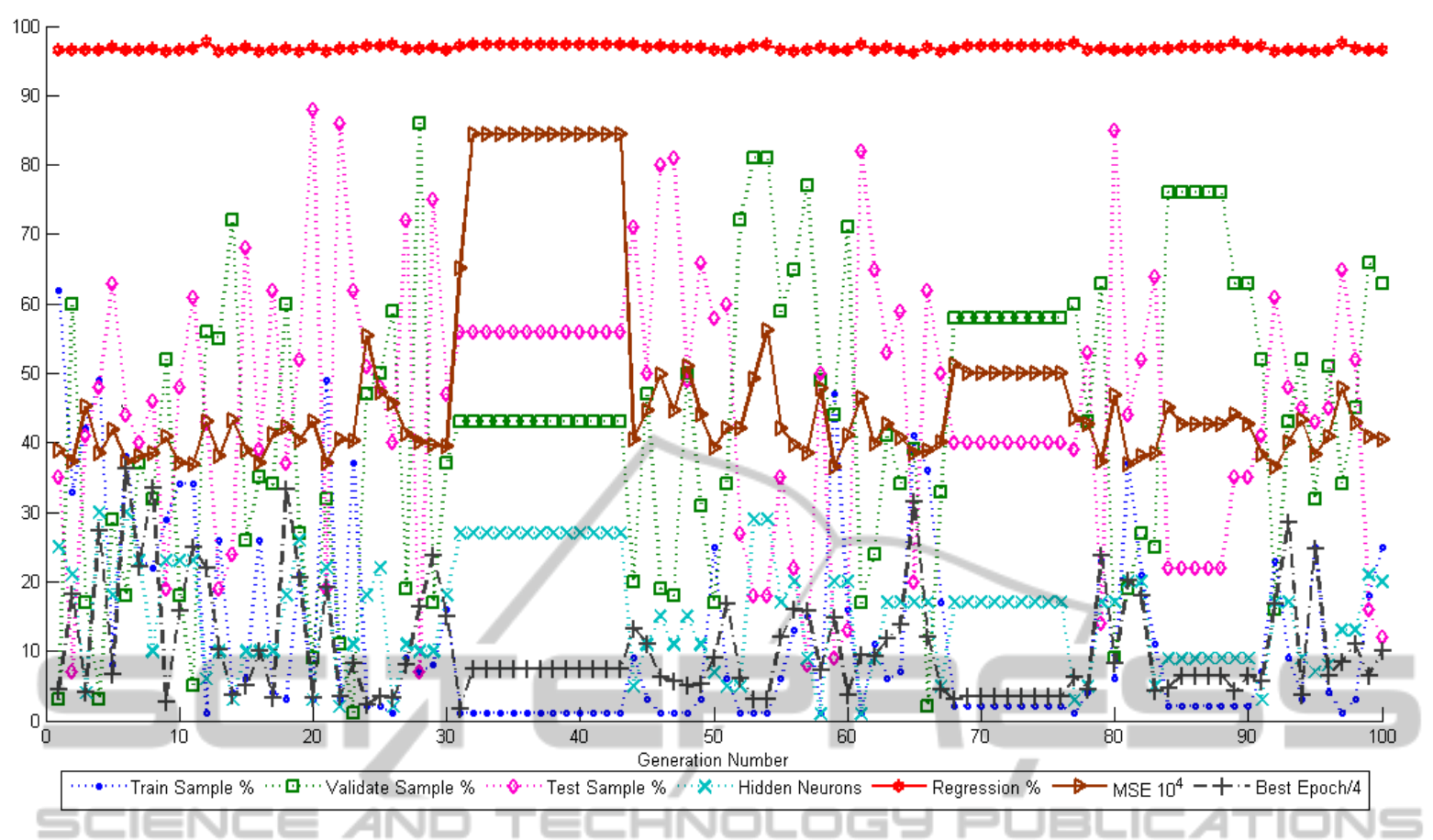

Figure 2: The evolution of the best performed chromosome of each generation (GA3).

and labelling them was possible as normal and attack data were collected separately. The final fitness value 0.97682 , outperforms or similar to the GA applications presented in (Gong et al., 2005); (Haddadi et al., 2010); (Hua and Xiaofeng, 2008); (Xia et al., 2005), where the results have obtained using sample traffic databases or synthetic traffic. This complete research was performed in a real environment, i.e. capturing of training data (used for training, validation and testing of $\mathrm{NN}$ ) and simulation data were from a real home WLAN. However, the GA never reached the $\mathrm{R}$ value obtained in (Ratnayake et al., 2011). Population of trainVal, validVal, and testVal and NeuL1 were between 1-62, 1-86, 7-88 and 1-30 respectively, whereas (Ratnayake et al., 2011) used 70, 15, 15 and 20 respectively (Table 1). Hence, although all the regression results are very close to global optimality and ideal fitness value of 1 , it is reasonable to suggest that the GA could be further improved.

\section{CONCLUSIONS}

IEEE 802.11 is the standard for WLAN communication. Attackers manipulate the requestresponse design vulnerability and send a flood of probe request frames which can cause serious performance degradation or prevent legitimate users from accessing network resources.

The research investigates and analyses WLAN traffic captured on a typical home wireless network, and uses supervised feedforward neural network with 4 input neurons, one hidden layer and an output neuron to determine the results. Empirical results show that all models perform similarly well.

The research utilised a GA to optimise structure of the neural network. The paper presents the process of systematic improvement of the GA and issues occurred during implementation. The regression value of the final GA was 0.97682 , which outperforms or similar to current research in the field of WIDS. However, GA did not reach the R value 0.98043 obtained in Ratnayake et al. (2011) as it never simulated with exact combination of $\mathrm{NN}$ structural values. However, the minimum regression value 0.96101 suggests that this NN model can be applied to unseen data successfully. The computer simulation results and optimisation results demonstrated that this approach helps the $\mathrm{NN}$ designer to find an optimised and reliable $\mathrm{NN}$ model using GAs without searching for every conceivable combination. Further research will be conducted in the future to enhance this experiment to optimise the $\mathrm{NN}$ training function. 


\section{REFERENCES}

Baker, J. E., (1987). Reducing bias and inefficiency in the selection algorithm. 2nd International Conference on Genetic Algorithms on Genetic algorithms and their application, MIT, Massachusetts.

Bankovic, Z., Stepanovic, D., Bojanic, S., and NietoTaladriz, O., (2007). Improving network security using genetic algorithm approach. Computers and Electrical Engineering, 33(5-6), 438-451. doi: 10.1016/j.compeleceng.2007.05.010.

De Jong, K. A., (1993). Genetic algorithms are NOT function optimizers. FOGA-92, 2nd workshop on Foundations of Genetic Algorithms, Vail, Colorado.

Gong, R. H., Zulkernine, M., and Abolmaesumi, P. (2005). A software implementation of a genetic algorithm based approach to network intrusion detection. Sixth International Conference on Software Engineering, Artificial Intelligence, Networking and Parallel/Distributed Computing, and First ACIS International Workshop on Self-Assembling Wireless Networks., 246-253. doi: 10.1109/SNPDSAWN.2005.9.

Haddadi, F., Khanchi, S., Shetabi, M., and Derhami, V. (2010). Intrusion Detection and Attack Classification Using Feed-Forward Neural Network. Second International Conference on Computer and Network Technology, Bangkok.

Holland, J. H., (1975). Adaptation in natural and artificial systems : an introductory analysis with applications to biology, control, and artificial intelligence. Ann Arbor, Mich.: University of Michigan Press.

Hua, J., and Xiaofeng, Z., (2008). Study on the network intrusion detection model based on genetic neural network. International Workshop on Modelling, Simulation and Optimization, Hong Kong.

Karygiannis, T., and Owens, L., (2002). Wireless network security. NIST special publication, 800, 48.

Landau, L. G., and Taylor, J. G., (1997). Concepts for neural networks: A survey: Springer-Verlag New York, Inc.

Levine, D., (1997). Commentary-Genetic Algorithms: A Practitioner's View. INFORMS Journal on Computing, 9(3), 256-259.

Li, W., (2004). Using genetic algorithm for network intrusion detection. United States Department of Energy Cyber Security Group 2004 Training Conference, Kansas City, Kansas.

Lindley, D. V., (1987). Regression and correlation analysis. New Palgrave: A Dictionary of Economics, 4, 120-123. doi: 10.1057/9780230226203.3411.

MathWorks, (2012). Post-Training Analysis (Network Validation): Multilayer Networks and Backpropagation Training (Neural Network Toolbox ${ }^{\mathrm{TM}}$ ) Retrieved 10 May, 2012, from http://www.mathworks.co.uk/help/toolbox/nnet/ug/bss 3318-1.html.

Moore, A. W., (2001). Cross-validation for detecting and preventing overfitting Retrieved 10 May, 2012, from http://www.autonlab.org/tutorials/overfit10.pdf.
Pillai, M. M., Eloff, J. H. P., and Venter, H. S., (2004). An approach to implement a network intrusion detection system using genetic algorithms. South African Institute for Computer Scientists and Information Technologists on IT research in developing countries, Republic of South Africa.

Ratnayake, D., Kazemian, H., Yusuf, S., and Abdullah, A., (2011). An Intelligent Approach to Detect Probe Request Attacks in IEEE 802.11 Networks. Engineering Applications of Neural Networks, Corfu, Greece.

Reeves, C. R., and Rowe, J. E. (2003). Genetic algorithms: principles and perspectives: a guide to $G A$ theory (Vol. 20): Springer.

Wu, S. X., and Banzhaf, W., (2010). Review: The use of computational intelligence in intrusion detection systems: A review. Appl. Soft Comput., 10(1), 1-35. doi: 10.1016/j.asoc.2009.06.019.

Xia, T., Qu, G., Hariri, S., and Yousif, M., (2005). An efficient network intrusion detection method based on information theory and genetic algorithm. 24th IEEE International Performance, Computing, and Communications Conference, 2005, Phoenix, Arizona.

Yao, X. H., (2010). A network intrusion detection approach combined with genetic algorithm and back propagation neural network. International Conference on E-Health Networking, Digital Ecosystems and Technologies, Shenzhen.

Zhang, Y., Zheng, J., and Ma, M., (2008). Handbook of research on wireless security: Information Science Reference-Imprint of: IGI Publishing. 